\title{
Violacein: Properties and Production of a Versatile Bacterial Pigment
}

\author{
Seong Yeol Choi, ${ }^{1}$ Kyoung-hye Yoon, ${ }^{2}$ Jin Il Lee, ${ }^{2}$ and Robert J. Mitchell ${ }^{1}$ \\ ${ }^{1}$ School of Life Sciences, Ulsan National Institute of Science and Technology, 50 UNIST-gil, Eonyang-eup, \\ Ulsan 689-798, Republic of Korea \\ ${ }^{2}$ Division of Biological Science and Technology, College of Science and Technology, Yonsei University, 1 Yonseidae-gil, \\ Wonju, Gangwon-do 220-710, Republic of Korea \\ Correspondence should be addressed to Jin Il Lee; jinillee@yonsei.ac.kr and Robert J. Mitchell; esgott@unist.ac.kr
}

Received 19 September 2014; Accepted 18 December 2014

Academic Editor: Flavia Marinelli

Copyright (C) 2015 Seong Yeol Choi et al. This is an open access article distributed under the Creative Commons Attribution License, which permits unrestricted use, distribution, and reproduction in any medium, provided the original work is properly cited.

Violacein-producing bacteria, with their striking purple hues, have undoubtedly piqued the curiosity of scientists since their first discovery. The bisindole violacein is formed by the condensation of two tryptophan molecules through the action of five proteins. The genes required for its production, vioABCDE, and the regulatory mechanisms employed have been studied within a small number of violacein-producing strains. As a compound, violacein is known to have diverse biological activities, including being an anticancer agent and being an antibiotic against Staphylococcus aureus and other Gram-positive pathogens. Identifying the biological roles of this pigmented molecule is of particular interest, and understanding violacein's function and mechanism of action has relevance to those unmasking any of its commercial or therapeutic benefits. Unfortunately, the production of violacein and its related derivatives is not easy and so various groups are also seeking to improve the fermentative yields of violacein through genetic engineering and synthetic biology. This review discusses the recent trends in the research and production of violacein by both natural and genetically modified bacterial strains.

\section{Natural Violacein-Producing Strains and Their Locales Are Quite Diverse}

As a bisindole, violacein (Figure 1) is produced by diverse genera of bacterial strains, including Collimonas [1], Duganella [2], Janthinobacterium [3-5], Microbulbifer sp. [6], and Pseudoalteromonas [7-9]. These violacein producers are varied phylogenetically and so are the locales from which they have been isolated. As shown in Table 1, these include quite a selection of environs as these bacteria have been found associated with the surfaces of sea sponges [7] and the rhizosphere of olive groves [10] and even within glaciers $[4,11,12]$. Perhaps the best known genus, however, is Chromobacterium $[13,14]$, which includes the strain C. violaceum [15].

\section{Violacein as an Indicator of Quorum Sensing}

In most of violacein-producing bacterial strains isolated from nature, this bisindole is a secondary metabolite that is associated with biofilm production [5]. Moreover, its production within C. violaceum and other strains is regulated by quorum sensing mechanisms [16]. Because it is easy to visualize, violacein production by $C$. violaceum has become a useful indicator of quorum sensing molecules and their inhibitors [17-20].

Secondary metabolites often serve functions other than the bacteria's immediate needs in growth and propagation. Many of these molecules are biologically active, and some have toxic properties to competing species giving the bacteria 
TABLE 1: Some violacein-producing strains and the locales from which they were isolated.

\begin{tabular}{lcc}
\hline Strain & Locale & Reference \\
\hline Chromobacterium violaceum & $\begin{array}{c}\text { River } \\
\text { Waste water } \\
\text { treatment plant }\end{array}$ & {$[14]$} \\
Collimonas sp. & $\begin{array}{c}\text { Arctic coastal } \\
\text { waters }\end{array}$ & {$[1]$} \\
Duganella sp. & $\begin{array}{c}\text { Agricultural soils } \\
\text { (olive) }\end{array}$ & {$[10]$} \\
D. violaceinigra & Forest soils & {$[22]$} \\
Janthinobacterium lividum & Glacier & {$[4]$} \\
J. svalbardensis & Glacier & {$[11]$} \\
Pseudoalteromonas sp. & Deep sea waters & {$[23]$} \\
P. luteoviolacea & (320 m) & {$[7]$} \\
\hline
\end{tabular}

a competitive advantage. Because of these activities, many secondary metabolites have been found to have pharmacological properties and, thus, are of interest for clinical use. In the first half of this review, therefore, we will review some of the biological functions, clinical significance, and mechanisms of action of violacein.

\section{Violacein's Function in Nature-Predator Defense?}

As noted above, violacein-producing bacteria encompass various genera and are found in all types of natural environments, from marine to freshwater and soil environments (Table 1). For this reason, it is rather difficult to pinpoint violacein's main biological or ecological role. One possible commonality is that violacein producers are generally sessile bacteria which makes them more vulnerable to predation [6]. This leads to the idea that violacein serves as some sort of defense mechanism for its producing strain or that it provides these sessile bacteria some competitive advantage.

Consistent with this, violacein was shown to have antibacterial properties particularly towards Gram-positive bacterial strains [36, 37]. One strain in particular that has received most of the recent attention is Staphylococcus aureus, in part due to its status as a multidrug resistant pathogen [37-40]. Two studies showed that crude violacein was capable of inhibiting $S$. aureus growth at concentrations between 5.7 and $15 \mathrm{mg} / \mathrm{L}$, or approximately 17 to $43 \mu \mathrm{mol} / \mathrm{L}[38,40]$.

Violacein's biological activity is not limited to prokaryotes, however, as it is also known to have negative effects on bacteriovorus protozoans and metazoans (Table 2). Matz et al. tested purified violacein and violacein-producing biofilms with various protozoan species that include flagellates, ciliates, and amoeba [6]. They showed that, when exposed to violacein, the bacteriovorus amoeba Acanthamoeba castellanii displays decreased feeding accompanied by morphological changes, such as cell rounding. Further observations, such as increases in caspase-3-like activity and TUNEL assay studies, suggest that these predacious microbes are dying via an apoptotic-like cell death [41].

Like many protists, the soil nematode C. elegans is a bacteriovorus predator that ingests a wide variety of microorganisms [42]. Although C. elegans is best known as a premier genetic model organism that has propelled such important discoveries as RNAi, microRNAs, and programmed cell death [43-45], it is also an excellent tool to understand violacein's biological activity on bacterial predators. For example, $C$. elegans fed $E$. coli as a prey strain grows normally and healthily in the laboratory. However, two strains of bacteria that produce violacein, Janthinobacterium sp. HH01 and C. violaceum, were shown to be toxic to C. elegans $[27,46]$. In particular $\mathrm{HH} 01$ exposures resulted in developmental problems in juvenile worms, as well as behavioral changes and a rapid mortality in adult worms. Interestingly, an exposure to a mutant HH01 strain that lacks violacein and tryptophan production did not induce severe toxic effects, indicating that the responses originated with the bisindole. As further confirmation of this, E. coli carrying HH01 vioA-E genes that produce violacein resulted in accelerated death, albeit at a slower rate than in HH01. These experiments affirm that violacein is likely toxic to the bacterial predator C. elegans.

In addition to its apparent roles in protecting the bacterium from predation, violacein seems to have other ecological functions that may provide advantages for the bacteria that produce it. For instance, violacein-producing Janthinobacterium forms biofilms and resides on the skins of frogs and salamanders [24, 47]. In return, the violacein produced provides antifungal protection for its amphibian hosts, increasing their survival.

\section{Potential Clinical Uses of Violacein}

These functional studies portray the secondary metabolite violacein as a toxic sentinel guarding against diverse potential bacterial predators and other competitors. However, such a broad cellular toxicity may also prove to be useful as a therapeutic against various pathogenic and endogenous cellular insults. Consistent with this, many pharmacological properties have been attributed to violacein.

Violacein has strong antibacterial effects making it a promising candidate as an antibiotic. Moreover, when administered in combination with other antibiotics, the impact is more effective in fighting bacteria than the use of antibiotics alone [48]. This is of particular interest in light of recent antibiotic-resistant strains of pathogenic bacteria, such as MRSA. Also, the antiprotozoan properties of violacein could be exploited to treat diseases in humans, such as in malaria and leishmaniasis $[25,26]$.

The most studied clinical use of violacein, however, is it being a potential cancer therapeutic. Violacein has been tested against various cancer cell lines (Table 3), where it has shown cytotoxicity at IC50 values that mostly range in the submicromolar concentrations. The effects of violacein were also shown to be specific for the cancer cell line tested as two colorectal cancer cell lines, Caco-2 and HT-29, were differentially susceptible to violacein [31]. Since violacein is cytotoxic towards noncancer cells as well $[28,34]$, the critical 
TABLE 2: Eukaryotic organisms in which violacein was shown to produce negative effects.

\begin{tabular}{|c|c|c|c|}
\hline Type & Organism & Description & Reference \\
\hline Fungi & Batrachochytrium dendrobatidis & Infects amphibians & {$[24]$} \\
\hline \multirow{6}{*}{ Protozoa } & Rhynchomonas nasuta & Flagellate & {$[6]$} \\
\hline & Tetrahymena sp. & Ciliate & {$[6]$} \\
\hline & Acanthamoeba castellanii & Amoeba & {$[6]$} \\
\hline & Leishmania amazonensis & Causative agent of leishmaniasis & {$[25]$} \\
\hline & Plasmodium falciparum & Causative agent of malaria in humans & {$[26]$} \\
\hline & Plasmodium chabaudi chabaudi & Causative agent of malaria in mice & {$[26]$} \\
\hline Nematode & Caenorhabditis elegans & & [27] \\
\hline
\end{tabular}

TABLE 3: List of cell lines tested against violacein.

\begin{tabular}{|c|c|c|c|c|}
\hline Cell line & Cell type & Organism & Notes & Reference \\
\hline V79 & Fibroblast-like cell line from lung tissue & Chinese Hamster & & {$[28]$} \\
\hline FRhK-4 & Fetal kidney & Monkey & & [29] \\
\hline Vero & Kidney & Monkey & & {$[29]$} \\
\hline MA104 & Kidney epithelial cells & Monkey & & {$[29]$} \\
\hline Hep2 & Hela-derived & Human & & [29] \\
\hline 92.1 & Uveal melanoma & Human & & {$[30]$} \\
\hline OCM-1 & Choroidal melanoma & Human & & {$[30]$} \\
\hline NCI-H460 & Non-small-cell lung cancer & Human & & {$[28]$} \\
\hline KM12 & Colon cancer & Human & & {$[28]$} \\
\hline Caco-2 & Heterogeneous epithelial colorectal adenocarcinoma & Human & & {$[31,32]$} \\
\hline НT29 & Colorectal adenocarcinoma & Human & & [31] \\
\hline HCT116 & Colorectal adenocarcinoma & Human & & {$[32]$} \\
\hline SW480 & Colorectal adenocarcinoma & Human & & {$[32]$} \\
\hline DLD1 & Colorectal adenocarcinoma & Human & & {$[32]$} \\
\hline TF1 & Erythroleukemia & Human & & {$[33]$} \\
\hline K562 & Lymphoma & Human & $\mathrm{N} / \mathrm{C}^{\mathrm{a}}$ & {$[34]$} \\
\hline U937 & Chronic myelogenic leukemia & Human & $\mathrm{N} / \mathrm{C}^{\mathrm{a}}$ & {$[34]$} \\
\hline HL60 & Promyelocytic leukemia & Human & & {$[34]$} \\
\hline MOLT-4 & Acute lymphoblastic leukemia & Human & & {$[28]$} \\
\hline EAT & Ehrlich ascites tumor & Mouse & In vivo ${ }^{\mathrm{b}}$ & {$[35]$} \\
\hline
\end{tabular}

${ }^{a}$ No cytotoxicity observed.

${ }^{\mathrm{b}}$ Both in vitro and in vivo tests were performed.

factor for its clinical use against cancer is that it is more toxic to cancer cells than to normal cells. One study demonstrated that violacein induced apoptosis in HL60 cells (IC50 = $700 \mathrm{nM}$ ), a cancer cell line used as a model to study myeloid leukemia. However, normal lymphocytes were unaffected at the concentrations tested, further asserting violacein's use as a putative cancer therapeutic [34].

Violacein clearly has toxic effects on cultured cancer cells, that is, within in vitro tests. Its ability to attenuate cancer growth in vivo was also tested recently in the Ehrlich ascites tumor (EAT) mouse model [35]. Daily intraperitoneal injections of $0.1 \mu \mathrm{g} / \mathrm{kg}$ violacein significantly increased the survival rate of the mice, while no adverse effects were observed in mice receiving higher doses of up to $1 \mathrm{mg} / \mathrm{kg}$.

\section{Biological Mechanism of Violacein's Effects}

Even though violacein is a promising agent as an antibiotic and a treatment for cancer, the biological mechanisms behind these actions remain elusive. Before we can stamp violacein as a natural therapeutic for numerous kinds of diseases, it is imperative to understand its mechanisms of action at the cellular and molecular levels.

Several groups have begun this and many of the studies performed in cancer cell lines report increased activity in indicators of apoptosis-related markers, such as increases in the reactive oxygen species (ROS) and the activation of caspases [31, 32, 34, 35]. However not all cancer cell lines respond to violacein and the reason for the selectivity is not 
well understood. Moreover, among the leukemia cell lines tested in the literature, violacein showed selective cytotoxicity against HL60 and TF1 (Table 3), but the pathways that lead to cell death were very different in the two cells. In HL60 cells, an exposure to violacein led to phosphorylation of p38 MAP kinase, upregulation of the $\mathrm{NF} \kappa \mathrm{B}$ pathway, and activation of caspases [34]. It was also found that TGF $\alpha$ receptor activation was required for these downstream effects. TF1 cells, on the other hand, did not seem to follow the canonical apoptotic pathway as treatment with inhibitors of proapoptotic caspases did not prevent cell death [33].

Conclusions on violacein's mechanism of action are clearly scant at this point. However, the fact that violacein has cytotoxic effects on such a wide variety of organisms and cells hints at a common target or pathway. Studying the effect of these bisindoles at the genetic level on model eukaryotic organisms, such as C. elegans, will help in elucidating its mechanism of action and enrich our knowledge of violacein as a clinical therapeutic. Owing to its versatile activity against many human ailments and infectious agents, however, it is not surprising that this bisindole has garnered more attention recently from the scientific community. One factor that may contribute to reducing violacein's application, though, is its relatively low level production by natural strains. Consequently, the latter half of this review will be primarily given to the discussion of current research into the production and purification of violacein and its related derivatives.

\section{Production of Violacein by Natural Host Strains}

Since violacein is produced naturally by various bacterial species, the use of these strains for its production seemed like a clear choice. However, many factors were found to influence the yields, including the agitation and aeration [7], the inoculum size $[2,10]$, and the nutrients available $[2,10,49,50]$. It should be noted that the violacein concentrations reported within many of the articles are based upon the extinction coefficient as determined by the authors using spectrophotometric analyses, with extinction coefficient values ranging between 10.955 and $74.3 \mathrm{~L} /(\mathrm{g}-\mathrm{cm})$ in the literature $[2,49$, 51, 52]. A recent article by Rodrigues et al. highlighted the discrepancy caused by spectrophotometric-based determinations of violacein and deoxyviolacein concentrations and stated that this could result in violacein concentrations that are inflated by as much as $680 \%$ [51]. To address this in their study, therefore, Rettori and Durán (1998) relied on HPLC measurements, a technique which was proposed in an earlier study to be used in parallel with NMR, UV-Vis spectroscopy, and mass spectroscopy when characterizing violacein and its production by bacterial strains [52]. Consequently, to avoid any potential confusion to the readers, this report will provide the violacein and deoxyviolacein concentrations reported and state whether they were determined via HPLC or with an extinction coefficient.

One group applied response surface methodologies (RSM) to identify the best conditions to produce violacein with C. violaceum [49]. They initially analyzed 16 variables but eventually limited them to three-glucose, tryptone, and yeast extract. While the latter two improved both the cell mass and violacein yields as they were increased, glucose was found to be negatively correlated with the violacein yields, and limiting its addition to the culture was advantageous. Using this technique, they were able to increase the dry cell weight (DCW) from 7.5 to $21 \mathrm{~g} / \mathrm{L}$ and the violacein yields from 170 to $430 \mathrm{mg} / \mathrm{L}$ with an extinction coefficient of $56.01 \mathrm{~L} /(\mathrm{g}$ $\mathrm{cm})$. Although there was a significant improvement in the volumetric productivity of violacein, defined as the $\mathrm{mg}$ of product per liter of culture, it should be noted that this does not represent a greater specific productivity of this bisindole, that is, mg product per gram of cells, as the cell mass increased by 2.8 -fold while the violacein concentration increased by only 2.5 -fold. Yet, similar protocols could potentially be employed with other strains to increase the cell density and, thus, raise the volumetric productivity.

A more recent study using RSM with another violaceinproducing strain, Pseudoduganella sp. B2, previously Duganella sp. B2, found similar results as the concentration of beef extract used was a major impetus for violacein production [2]. They also identified the culture $\mathrm{pH}$ and the concentrations of tryptophan and potassium nitrate as major players influencing the final violacein yields. The importance of tryptophan is not surprising as this is the precursor for violacein, while the impact of nitrate was thought to be related to nitrogen source availability for the growing bacterial cultures. Under the optimal conditions found, the authors claimed Duganella sp. B2 was capable of producing $1.6 \mathrm{~g} / \mathrm{L}$ crude violacein, typically referring to the naturally produced mixture of violacein and deoxyviolacein. This value is under scrutiny, however, as the extinction coefficient used $(10.955 \mathrm{~L} /$ $(\mathrm{g}-\mathrm{cm}))$ is the lowest reported in the literature $[2,49,51,52]$.

\section{Production of Violacein within $E$. coli and Other Heterologous Hosts}

Since the genes required for the production of violacein are known to exist within a single operon, that is, vioABCDE [53], many groups have sought to clone and express these within other bacterial hosts, including E. coli $[8,51,53]$. The Pemberton group has focused on plasmid stability, an issue when trying to generate bacterial products in long term and in nonnatural hosts. They found, for instance, when the violacein gene cluster was cloned into $\mathrm{pHC79}$, a cosmid vector, that it was unstable and was lost in as much as $60 \%$ of the bacterial population when grown for 15 generations in the absence of antibiotic pressure [54]. They claimed that the same was true when they expressed the vio $A B C D E$ operon in a pUC18 vector but were able to generate a stable construct using a broad host range IncP plasmid [54, 55]. This plasmid, pPSX-Vio ${ }^{+}$, was stable without antibiotics for more than 100 generations [54], making it a potentially useful tool for the production of violacein.

In a subsequent study, they found that the amount of violacein produced by E. coli was dependent upon the host, with $E$. coli strain JM109 producing 3.9-fold more violacein than E. coli strain DH5 $\alpha$ when harboring the same plasmid [55]. The production of the alpha amylase protein, AmyA, from Streptomyces lividans was likewise found to be better in 


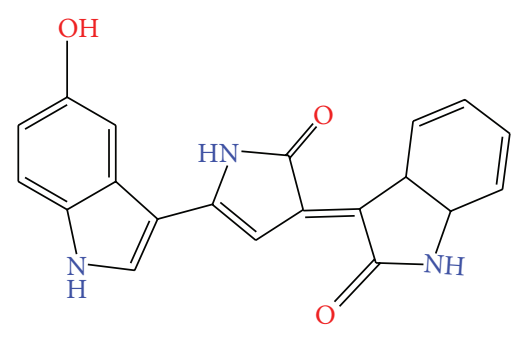

Violacein

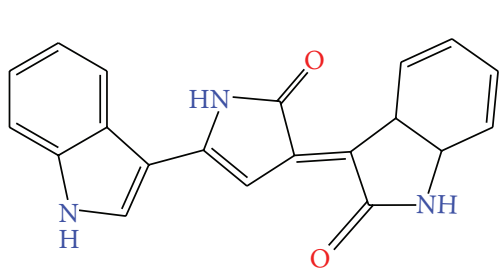

Deoxyviolacein

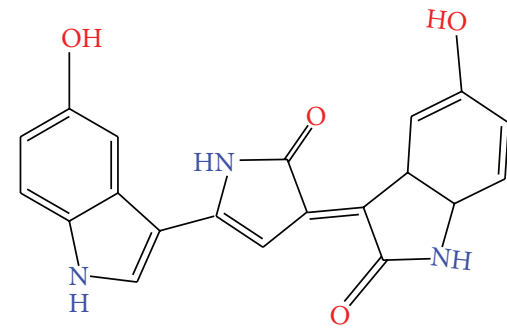

Oxyviolacein

FIGURE 1: Structures of violacein, deoxyviolacein, and oxyviolacein showing either the presence or the lack of the hydroxyl groups. The structures were prepared using ChemDoodle 2D sketcher (http://web.chemdoodle.com/demos/sketcher).

JM109 than DH5 $\alpha$, demonstrating that this phenotype was characteristic of JM109 and not due to the violacein genes. The authors attributed better results with JM109 to the genetic differences between the host strains but did not delve deeper into the mechanisms underlying this phenotype. However, they did identify a mutation within the plasmid which led to a further enhancement in the violacein yields [55]. This mutation, which the authors designated as opv-1 (overproduction of violacein), results from the deletion of a single nucleotide within the region upstream of the vioA gene, leading to a 4.2- and 2.9-fold enhanced violacein yield from E. coli strains DH5 $\alpha$ and JM109, respectively.

A more recent study sought to engineer $E$. coli and its metabolic pathways to improve the violacein yields [51]. For this, the authors overexpressed the genes related to tryptophan production and knocked out several genes and pathways which would detract the carbon flux away from this amino acid. The engineered E. coli strain, TRP11, produces about $20 \mu \mathrm{mol}$ tryptophan per gram DCW (gDCW). By comparison, the control wild-type strain only produced about $0.3 \mu \mathrm{mol}$ tryptophan/gDCW, representing an increase in the tryptophan concentration of more the 60 -fold. They next introduced the vioD gene into the chromosome of this strain and a plasmid expressing the vioABCE genes. Performing fedbatch fermentations over a 12-day period with this strain, which they designated as Vio-4, they were able to generate $710 \mathrm{mg} / \mathrm{L}$ violacein at more than $99 \%$ purity, demonstrating that $E$. coli can be used to produce high level concentrations of this bisindole specifically. E. coli, however, has not been the only strain to have been used as a host to produce the bisindole violacein. Both Citrobacter freundii and Enterobacter aerogenes have also been used with positive results [56].

\section{Deoxyviolacein and Oxyviolacein}

Although violacein has received most of the attention, it is not the only compound being produced by the VioA, VioB, VioC, VioD, and VioE proteins within these bacterial hosts. A recent article that describes the various products in detail was recently published and lists over a dozen different compounds that have been produced in tests with various strains, mutants, and cell-free extracts [13]. Perhaps the best known derivative produced by the violacein biosynthetic pathway, however, is deoxyviolacein. In J. lividum, for example, deoxyviolacein (Figure 1) is also being produced, albeit at a lower level than violacein [57]. Similar results were also seen with Duganella sp. B2 where the production of deoxyviolacein was lower than that of violacein, as measured by HPLC [58]. The percent deoxyviolacein within the crude violacein extracts obtained from the natural bacterial hosts is typically around $10 \sim 20 \%$, with the vast majority being violacein. This is also true for commercially available violacein from Sigma-Aldrich, which is prepared using J. lividum and certified as at least $85 \%$ violacein based upon HPLC analysis.

Deoxyviolacein, which lacks a hydroxyl group (Figure 1), can be produced by omitting the VioD protein. Using a recombinant Citrobacter freundii carrying a plasmid with a knock-out in the vioD gene, a recent study by the Xing group reported on the high level production and characterization of deoxyviolacein [58], with an emphasis given to the differences between violacein and deoxyviolacein. Their study showed, for example, that the photostability of deoxyviolacein was slightly better than violacein in tests with either natural or UV light. Moreover, in 24-hour toxicity tests with HepG2 cell lines, violacein and deoxyviolacein were both found to be toxic. However, the impact of violacein was dosedependent as greater additions of this bisindole led to greater concomitant losses in the HepG2 viability. In contrast, the toxicity of deoxyviolacein was not dose-dependent but led to fairly consistent and stable losses in the viability over a range of concentrations $(0.1-10 \mu \mathrm{M})$. The difference between these two bisindoles was even more pronounced when the viability was determined after 48 hours.

Several groups have also shown that the proteins responsible for the biosynthesis of violacein are not strict for their typical substrate, tryptophan, as 5-hydroxytryptophan can also be used to produce another derivative, oxyviolacein (Figure 1) $[59,60]$. In contrast to deoxyviolacein which lacks a hydroxyl group when compared with violacein, oxyviolacein boasts an additional hydroxyl group. Likewise, as the loss of the hydroxyl group in deoxyviolacein reduced its efficacy against $S$. aureus, the presence of this extra hydroxyl group within oxyviolacein was found to increase the potency against these human pathogens [61].

\section{Conclusions}

This review presents current research trends regarding the study and production of the bacterially produced bisindole violacein and several derivatives. As a secondary metabolite, 
violacein has been found to possess a wide variety of biological activities, including anticancerous properties. These characteristics have led to renewed interest in this compound and its production by both wild-type and recombinant bacterial strains. As presented in this report, the production and characterization of violacein are not without their own obstacles and struggles, and much work still needs to be done. This is particularly true regarding the mode of activity of violacein which needs to be studied more in depth. Current trends in molecular genetics are aiding in this as researchers are now capable of engineering bacterial host that can overproduce this bisindole within fermentations. As work with this compound and its derivatives progresses, it is anticipated that violacein will become more readily available for the scientific community and clinical studies.

\section{Conflict of Interests}

The authors declare that there is no conflict of interests regarding the publication of this paper.

\section{Authors' Contribution}

Seong Yeol Choi and Kyoung-hye Yoon contributed equally to this paper. All authors participated in preparation of this paper.

\section{Acknowledgments}

Seong Yeol Choi and Robert J. Mitchell received support from the Korea Institute for Advancement of Technology under the EUREKA Programme (Grant no. N019800009) and the Korea Health Industry Development Institute (KHIDI) (Grant no. HI13C13550000). Jin Il Lee and Kyoung-hye Yoon are supported by a New Investigator Grant from the National Research Foundation of Korea (Grant no. 201405553). The authors appreciate the support.

\section{References}

[1] S. Hakvåg, E. Fjærvik, G. Klinkenberg et al., "Violacein-producing Collimonas sp. from the sea surface microlayer of costal waters in Trøndelag, Norway," Marine Drugs, vol. 7, no. 4, pp. 576-588, 2009.

[2] H. S. Wang, P. X. Jiang, Y. Lu et al., "Optimization of culture conditions for violacein production by a new strain of Duganella sp. B2," Biochemical Engineering Journal, vol. 44, no. 2-3, pp. 119124, 2009.

[3] B. A. Jude, J. Tanner, T. Koko, and E. C. McLaughlin, "Analysis, characterization, and synthesis of violacein from Janthinobacterium isolate extracts," Abstracts of Papers of the American Chemical Society, vol. 244, 2012.

[4] Y. Lu, L. Wang, Y. Xue et al., "Production of violet pigment by a newly isolated psychrotrophic bacterium from a glacier in Xinjiang, China," Biochemical Engineering Journal, vol. 43, no. 2, pp. 135-141, 2009.

[5] F. Pantanella, F. Berlutti, C. Passariello, S. Sarli, C. Morea, and S. Schippa, "Violacein and biofilm production in Janthinobacterium lividum," Journal of Applied Microbiology, vol. 102, no. 4, pp. 992-999, 2007.
[6] C. Matz, J. S. Webb, P. J. Schupp et al., "Marine biofilm bacteria evade eukaryotic predation by targeted chemical defense," PLoS ONE, vol. 3, no. 7, Article ID e2744, 2008.

[7] L. H. Yang, H. Xiong, O. O. Lee, S.-H. Qi, and P.-Y. Qian, "Effect of agitation on violacein production in Pseudoalteromonas luteoviolacea isolated from a marine sponge," Letters in Applied Microbiology, vol. 44, no. 6, pp. 625-630, 2007.

[8] X. Zhang and K. Enomoto, "Characterization of a gene cluster and its putative promoter region for violacein biosynthesis in Pseudoalteromonas sp. 520P1," Applied Microbiology and Biotechnology, vol. 90, no. 6, pp. 1963-1971, 2011.

[9] S. A. Mccarthy, R. M. Johnson, D. kakimoto, and T. sakata, "Effects of various agents on the pigment (violacein) and antibiotic production of Alteromonas luteoviolacea," Bulletin of the Japanese Society of Scientific Fisheries, vol. 51, no. 7, pp. 1115-1121, 1985.

[10] S. Aranda, M. Montes-Borrego, and B. B. Landa, "Purplepigmented violacein-producing Duganella spp. inhabit the rhizosphere of wild and cultivated olives in Southern Spain," Microbial Ecology, vol. 62, no. 2, pp. 446-459, 2011.

[11] J. A. Avguštin, D. Z. Bertok, R. Kostanjšek, and G. Avguštin, "Isolation and characterization of a novel violacein-like pigment producing psychrotrophic bacterial species Janthinobacterium svalbardensis sp. nov," Antonie van Leeuwenhoek, International Journal of General and Molecular Microbiology, vol. 103, no. 4, pp. 763-769, 2013.

[12] S. J. Kim, S. C. Shin, S. G. Hong et al., "Genome sequence of Janthinobacterium sp. strain PAMC 25724, isolated from alpine glacier cryoconite," Journal of Bacteriology, vol. 194, no. 8, pp. 2096-2096, 2012.

[13] T. Hoshino, "Violacein and related tryptophan metabolites produced by Chromobacterium violaceum: biosynthetic mechanism and pathway for construction of violacein core," Applied Microbiology and Biotechnology, vol. 91, no. 6, pp. 1463-1475, 2011.

[14] M. O. Moss, C. Ryall, and N. A. Logan, "The classification and characterization of chromobacteria from a lowland river," Journal of General Microbiology, vol. 105, no. 1, pp. 11-21, 1978.

[15] N. D. Durán and C. F. M. Menck, "Chromobacterium violaceum: a review of pharmacological and industiral perspectives," Critical Reviews in Microbiology, vol. 27, no. 3, pp. 201-222, 2001.

[16] K. H. McClean, M. K. Winson, L. Fish et al., "Quorum sensing and Chromobacterium violaceum: exploitation of violacein production and inhibition for the detection of $N$-acylhomoserine lactones," Microbiology, vol. 143, no. 12, pp. 3703-3711, 1997.

[17] S. A. Burt, V. T. A. Ojo-Fakunle, J. Woertman, and E. J. A. Veldhuizen, "The natural antimicrobial carvacrol inhibits quorum sensing in Chromobacterium violaceum and reduces bacterial biofilm formation at sub-lethal concentrations," PLOS ONE, vol. 9, no. 4, Article ID e93414, 2014.

[18] P. S. Rajesh and V. Ravishankar Rai, "Quorum quenching activity in cell-free lysate of endophytic bacteria isolated from Pterocarpus santalinus Linn., and its effect on quorum sensing regulated biofilm in Pseudomonas aeruginosa PAO1," Microbiological Research, vol. 169, no. 7-8, pp. 561-569, 2014.

[19] J. C. Taganna, J. P. Quanico, R. M. G. Perono, E. C. Amor, and W. L. Rivera, "Tannin-rich fraction from Terminalia catappa inhibits quorum sensing (QS) in Chromobacterium violaceum and the QS-controlled biofilm maturation and LasA staphylolytic activity in Pseudomonas aeruginosa," Journal of Ethnopharmacology, vol. 134, no. 3, pp. 865-871, 2011. 
[20] M. Durán, A. Faljoni-Alario, and N. Durán, “Chromobacterium violaceum and its important metabolites-review," Folia Microbiologica, vol. 55, no. 6, pp. 535-547, 2010.

[21] W. A. Ahmad, N. Z. Yusof, N. Nordin, Z. A. Zakaria, and M. F. Rezali, "Production and characterization of violacein by locally isolated Chromobacterium violaceum grown in agricultural wastes," Applied Biochemistry and Biotechnology, vol. 167, no. 5, pp. 1220-1234, 2012.

[22] W. J. Li, Y. Q. Zhang, D. J. Park et al., "Duganella violaceinigra sp. nov., a novel mesophilic bacterium isolated from forest soil," International Journal of Systematic and Evolutionary Microbiology, vol. 54, part 5, pp. 1811-1814, 2004.

[23] S. Yada, Y. Wang, Y. Zou et al., "Isolation and characterization of two groups of novel marine bacteria producing violacein," Marine Biotechnology, vol. 10, no. 2, pp. 128-132, 2008.

[24] R. M. Brucker, R. N. Harris, C. R. Schwantes et al., "Amphibian chemical defense: antifungal metabolites of the microsymbiont Janthinobacterium lividum on the salamander Plethodon cinereus," Journal of Chemical Ecology, vol. 34, no. 11, pp. 14221429, 2008.

[25] L. L. Leon, C. C. Miranda, A. O. de Souza, and N. Durán, "Antileishmanial activity of the violacein extracted from Chromobacterium violaceum," Journal of Antimicrobial Chemotherapy, vol. 48, no. 3, pp. 449-450, 2001.

[26] S. C. P. Lopes, Y. C. Blanco, G. Z. Justo et al., "Violacein extracted from Chromobacterium violaceum inhibits Plasmodium growth in vitro and in vivo," Antimicrobial Agents and Chemotherapy, vol. 53, no. 5, pp. 2149-2152, 2009.

[27] C. Hornung, A. Poehlein, F. S. Haack et al., "The Janthinobacterium sp. HH01 Genome Encodes a Homologue of the $V$. cholerae CqsA and L. pneumophila LqsA Autoinducer Synthases," PLoS ONE, vol. 8, no. 2, Article ID e55045, 2013.

[28] P. da Silva Melo, S. S. Maria, B. de Campos Vidal, M. Haun, and N. Durán, "Violacein cytotoxicity and induction of apoptosis in V79 cells," In Vitro Cellular \& Developmental Biology-Animal, vol. 36, no. 8, pp. 539-543, 2000.

[29] C. R. Andrighetti-Fröhner, R. V. Antonio, T. B. CreczynskiPasa, C. R. M. Barardi, and C. M. O. Simões, "Cytotoxicity and potential antiviral evaluation of violacein produced by Chromobacterium violaceum," Memorias do Instituto Oswaldo Cruz, vol. 98, no. 6, pp. 843-848, 2003.

[30] V. S. Saraiva, J.-C. Marshall, J. Cools-Lartigue, and M. N. Burnier Jr., "Cytotoxic effects of violacein in human uveal melanoma cell lines," Melanoma Research, vol. 14, no. 5, pp. 421-424, 2004.

[31] D. D. de Carvalho, F. T. M. Costa, N. Duran, and M. Haun, "Cytotoxic activity of violacein in human colon cancer cells," Toxicology in Vitro, vol. 20, no. 8, pp. 1514-1521, 2006.

[32] L. L. Kodach, C. L. Bos, N. Durán, M. P. Peppelenbosch, C. V. Ferreira, and J. C. H. Hardwick, "Violacein synergistically increases 5-fluorouracil cytotoxicity, induces apoptosis and inhibits Akt-mediated signal transduction in human colorectal cancer cells," Carcinogenesis, vol. 27, no. 3, pp. 508-516, 2006.

[33] K. C. S. Queiroz, R. Milani, R. R. Ruela-de-Sousa et al., "Violacein induces death of resistant leukaemia cells via kinome reprogramming, endoplasmic reticulum stress and Golgi apparatus collapse," PLoS ONE, vol. 7, no. 10, Article ID e45362, 2012.

[34] C. V. Ferreira, C. L. Bos, H. H. Versteeg, G. Z. Justo, N. Durán, and M. P. Peppelenbosch, "Molecular mechanism of violaceinmediated human leukemia cell death," Blood, vol. 104, no. 5, pp. 1459-1464, 2004.
[35] N. Bromberg, J. L. Dreyfuss, C. V. Regatieri et al., "Growth inhibition and pro-apoptotic activity of violacein in Ehrlich ascites tumor," Chemico-Biological Interactions, vol. 186, no. 1, pp. 4352, 2010.

[36] H. C. Lichstein and V. F. van de Sand, "Violacein, an antibiotic pigment produced by Chromobacterium violaceum," Journal of Infectious Diseases, vol. 76, no. 1, pp. 47-51, 1945.

[37] Y. Nakamura, T. Sawada, Y. Morita, and E. Tamiya, "Isolation of a psychrotrophic bacterium from the organic residue of a water tank keeping rainbow trout and antibacterial effect of violet pigment produced from the strain," Biochemical Engineering Journal, vol. 12, no. 1, pp. 79-86, 2002.

[38] Y. Nakamura, C. Asada, and T. Sawada, "Production of antibacterial violet pigment by psychrotropic bacterium RT102 strain," Biotechnology and Bioprocess Engineering, vol. 8, no. 1, pp. 3740, 2003.

[39] N. G. Vynne, M. Mansson, and L. Gram, "Gene sequence based clustering assists in dereplication of Pseudoalteromonas luteoviolacea strains with identical inhibitory activity and antibiotic production," Marine Drugs, vol. 10, no. 8, pp. 1729-1740, 2012.

[40] S. Subramaniam, V. Ravi, and A. Sivasubramanian, "Synergistic antimicrobial profiling of violacein with commercial antibiotics against pathogenic micro-organisms," Pharmaceutical Biology, vol. 52, no. 1, pp. 86-90, 2014.

[41] P. D. Melo, S. S. Maria, B. D. Vidal, M. Haun, and N. Duran, "Violacein cytotoxicity and induction of apoptosis in V79 cells," In Vitro Cellular \& Developmental Biology-Animal, vol. 36, no. 8, pp. 539-543, 2000.

[42] S. Brenner, "The genetics of Caenorhabditis elegans," Genetics, vol. 77, no. 1, pp. 71-94, 1974.

[43] A. Fire, S. Xu, M. K. Montgomery, S. A. Kostas, S. E. Driver, and C. C. Mello, "Potent and specific genetic interference by doublestranded RNA in caenorhabditis elegans," Nature, vol. 391, no. 6669, pp. 806-811, 1998.

[44] R. C. Lee, R. L. Feinbaum, and V. Ambros, "The C. elegans heterochronic gene lin-4 encodes small RNAs with antisense complementarity to lin-14," Cell, vol. 75, no. 5, pp. 843-854, 1993.

[45] M. O. Hengartner and H. R. Horvitz, "Programmed cell death in Caenorhabditis elegans," Current Opinion in Genetics and Development, vol. 4, no. 4, pp. 581-586, 1994.

[46] L. R. Swem, D. L. Swem, C. T. O’Loughlin et al., "A quorumsensing antagonist targets both membrane-bound and cytoplasmic receptors and controls bacterial pathogenicity," Molecular Cell, vol. 35, no. 2, pp. 143-153, 2009.

[47] R. N. Harris, R. M. Brucker, J. B. Walke et al., "Skin microbes on frogs prevent morbidity and mortality caused by a lethal skin fungus," The ISME Journal, vol. 3, no. 7, pp. 818-824, 2009.

[48] S. Subramaniam, V. Ravi, and A. Sivasubramanian, "Synergistic antimicrobial profiling of violacein with commercial antibiotics against pathogenic micro-organisms," Pharmaceutical Biology, vol. 52, no. 1, pp. 86-90, 2014.

[49] A. S. Mendes, J. E. de Carvalho, M. C. T. Duarte, N. Durán, and R. E. Bruns, "Factorial design and response surface optimization of crude violacein for Chromobacterium violaceum production," Biotechnology Letters, vol. 23, no. 23, pp. 1963-1969, 2001.

[50] R. Riveros, M. Haun, and N. Durán, "Effect of growth conditions on production of violacein by Chromobacterium violaceum (BB-78 strain)," Brazilian Journal of Medical and Biological Research, vol. 22, no. 5, pp. 569-577, 1989.

[51] A. L. Rodrigues, N. Trachtmann, J. Becker et al., "Systems metabolic engineering of Escherichia coli for production of the 
antitumor drugs violacein and deoxyviolacein," Metabolic Engineering, vol. 20, pp. 29-41, 2013.

[52] D. Rettori and N. Durán, "Production, extraction and purification of violacein: an antibiotic pigment produced by Chromobacterium violaceum," World Journal of Microbiology and Biotechnology, vol. 14, no. 5, pp. 685-688, 1998.

[53] P. R. August, T. H. Grossman, C. Minor et al., "Sequence analysis and functional characterization of the violacein biosynthetic pathway from Chromobacterium violaceum," Journal of Molecular Microbiology and Biotechnology, vol. 2, no. 4, pp. 513-519, 2000.

[54] D. S. Sarovich and J. M. Pemberton, "pPSX: a novel vector for the cloning and heterologous expression of antitumor antibiotic gene clusters," Plasmid, vol. 57, no. 3, pp. 306-313, 2007.

[55] A. Ahmetagic and J. M. Pemberton, "Stable high level expression of the violacein indolocarbazole anti-tumour gene cluster and the Streptomyces lividans amyA gene in E. coli K12," Plasmid, vol. 63, no. 2, pp. 79-85, 2010.

[56] P.-X. Jiang, H.-S. Wang, C. Zhang, K. Lou, and X.-H. Xing, "Reconstruction of the violacein biosynthetic pathway from Duganella sp. B2 in different heterologous hosts," Applied Microbiology and Biotechnology, vol. 86, no. 4, pp. 1077-1088, 2010.

[57] A. L. Rodrigues, Y. Göcke, C. Bolten, N. L. Brock, J. S. Dickschat, and C. Wittmann, "Microbial production of the drugs violacein and deoxyviolacein: analytical development and strain comparison," Biotechnology Letters, vol. 34, no. 4, pp. 717-720, 2012.

[58] P.-X. Jiang, H.-S. Wang, S. Xiao et al., "Pathway redesign for deoxyviolacein biosynthesis in Citrobacter freundii and characterization of this pigment," Applied Microbiology and Biotechnology, vol. 94, no. 6, pp. 1521-1532, 2012.

[59] C. Sánchez, A. F. Braña, C. Méndez, and J. A. Salas, "Reevaluation of the violacein biosynthetic pathway and its relationship to indolocarbazole biosynthesis," ChemBioChem, vol. 7, no. 8, pp. 1231-1240, 2006.

[60] T. Hoshino and N. Ogasawara, "Biosynthesis of violacein: evidence for the intermediacy of 5-hydroxy-L-tryptophan and the structure of a new pigment, oxyviolacein, produced by the metabolism of 5-hydroxytryptophan," Agricultural and Biological Chemistry, vol. 54, no. 9, pp. 2339-2346, 1990.

[61] H. S. Wang, F. Z. Wang, X. F. Zhu et al., "Biosynthesis and characterization of violacein, deoxyviolacein and oxyviolacein in heterologous host, and their antimicrobial activities," Biochemical Engineering Journal, vol. 67, pp. 148-155, 2012. 

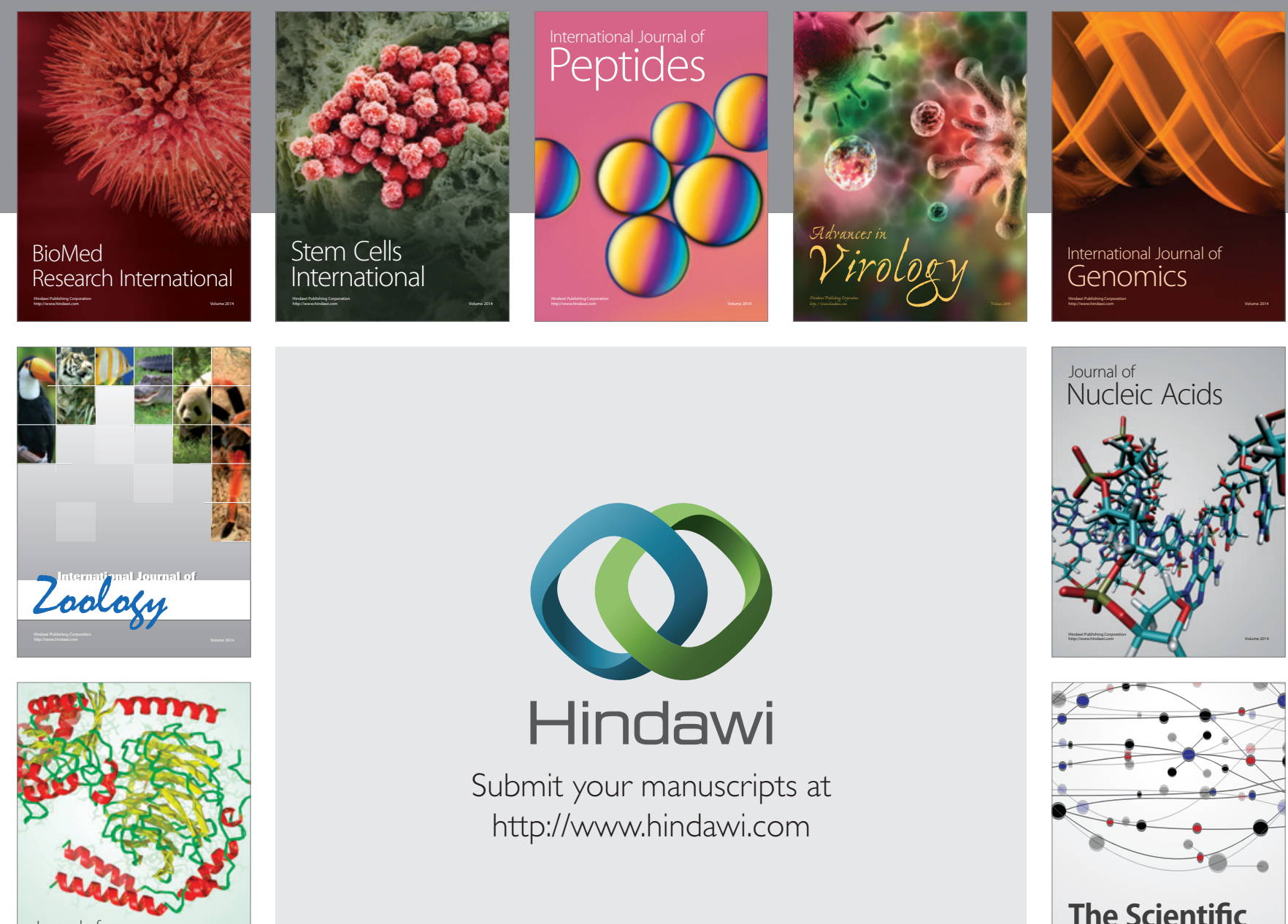

Submit your manuscripts at

http://www.hindawi.com

Journal of
Signal Transduction
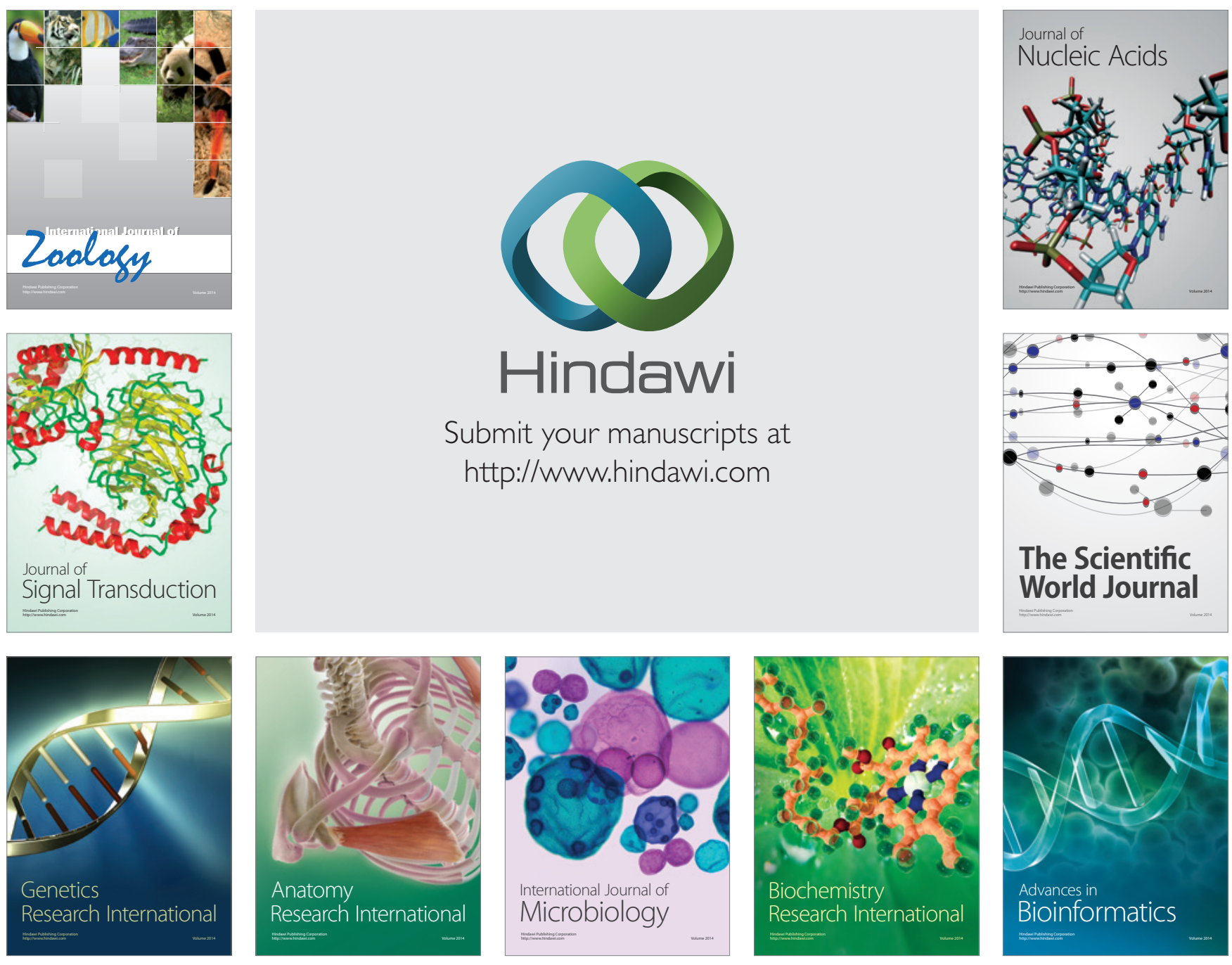

The Scientific World Journal
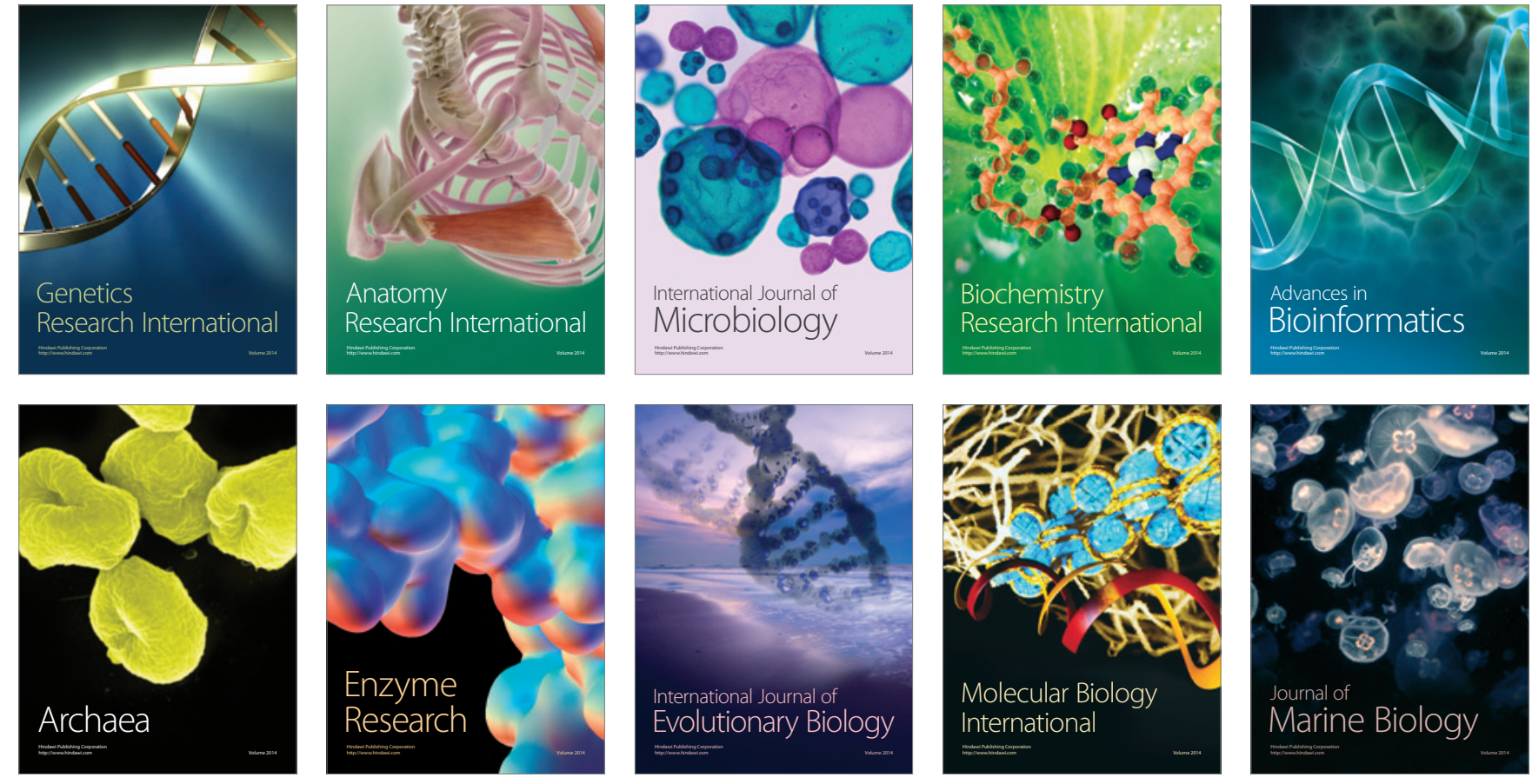